\title{
RFID Überwachungssystem für rotierende Maschinenelemente
}

\author{
Dipl.-Ing. Jörg Hübler, Staatlich geprüfter Techniker (FS) Tino Puggel, \\ Prof. Dr.-Ing. Klaus Nendel, Dipl.-Ing. Uwe Dombeck \\ Technische Universität Chemnitz \\ Institut für Fördertechnik und Kunststoffe \\ Professur Fördertechnik
}

\begin{abstract}
Maschinenelementen wie Kupplungen und Naben können Lebensdauer, Systemveränderungen und Ausfälle eines Bauteils frühzeitig erkennen und damit den gesamten Prozessablauf sicherer und effizienter gestalten. Wartungsintervalle können dem realen Verschleiß des Bauteils angepasst oder Überlastungen frühzeitig erkannt werden.
\end{abstract}

Den Ansatzpunkt zur Umsetzung liefert die RFID Technik. Bestehend aus Transponder, Lesegerät und entsprechenden Sensoren kann diese Technik nicht nur zur Lokalisierung und Identifizierung, sondern auch zur drahtlosen Überwachung von Bauteilen eingesetzt werden.

Unter Verwendung der RFID Technik wurde ein passives Transpondersystem entwickelt, dass verschiedene Sensoren zur Zustandsüberwachung erfassen kann. Dabei spielte die Energiebilanz des Systems eine bedeutende Rolle, um die Transponderversorgung durch ein äußeres elektrisches Feld möglichst einfach zu gestalten. Während des Projektes entstand ein Prototyp eines Überwachungssystems für rotierende Maschinenelemente, an dem in zahlreichen Tests in Verbindung mit Stahllamellenkupplungen die geforderten Funktionsfähigkeiten nachgewiesen wurden. Winkelversatz und der Zustand der Lamelle (Bruch) konnten dabei permanent überwacht werden.

\section{$1 \quad$ Einleitung}

Stahl-Lamellenkupplungen (Abbildung 1: Stahllamellenkupplungen werden nahezu in allen bedeutenden nationalen und internationalen Unternehmen aus den Bereichen Hydraulik, Maschinen- und Anlagenbau vielfach eingesetzt.

Die Verwendung von Stahllamellenkupplungen erfordert eine genaue Einhaltung der zulässigen Winkel- bzw. Radialverlagerung der beiden zu verbindenden Wellenenden. In der Praxis kommt es jedoch häufig vor, dass sich einzelne Aggregate in ihrer Lage zueinander verändern und die maximal zulässigen Winkelund Radialabweichungen dabei überschreiten. Werden diese Kupplungen dann weiter betrieben, kommt es zum Lamellenbruch und kurze Zeit später zu Funktionseinschränkungen bis zum Ausfall.

Eine Überwachung und Bewertung der Ist-Zustandsdaten während des Betriebes wird daher angestrebt. Die ermittelten Daten können ebenso zur Lebensdauerabschätzung und Wartung des Kupplungssystems verwendet werden. 


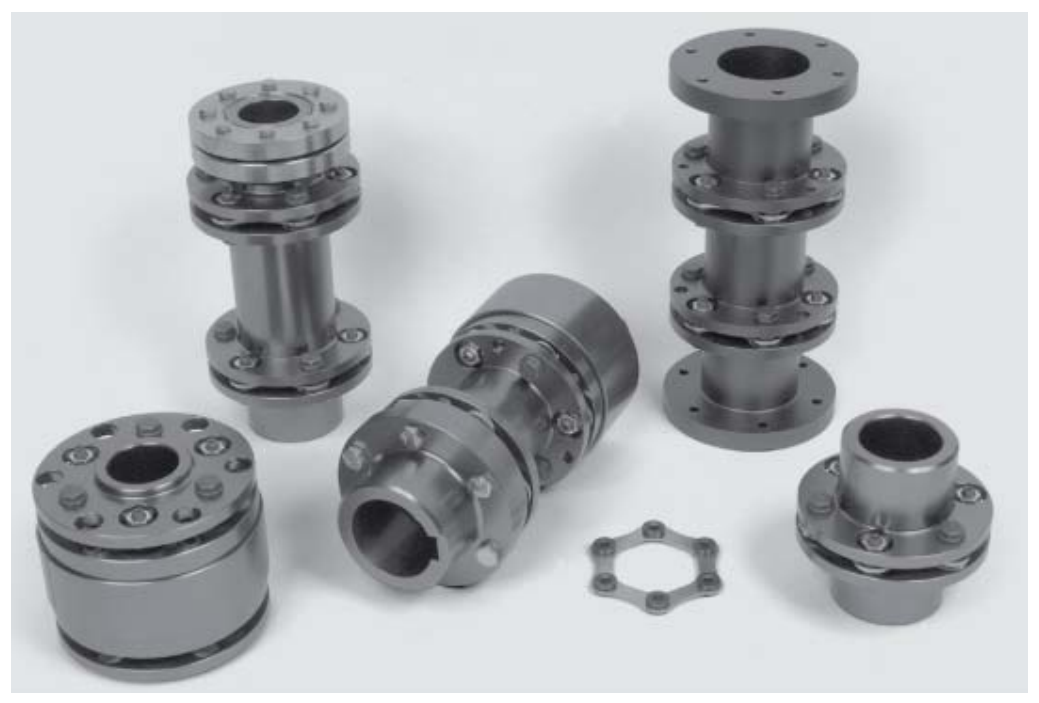

Abbildung 1: Stahllamellenkupplungen [Lov10]

\section{Theoretische Grundlagen}

Die RFID-Technologie (Radio_Frequency_IDentification) ermöglicht eine berührungslose Datenübertragung von Messwerten, die zwischen Transponder und Lesegerät ausgetauscht werden. Aufgebaut sind RFID-Systeme aus einem passiv, aktiv oder semi-aktiv arbeitenden Transponder, einem Lesegerät, betehend aus Sender, Empfänger und einer Antenne, dass mit einer Kontrolleinheit verbunden ist (Abbildung 2: RFID Funktionsprinzip [Fin08]). Die Kommunikation mit dem Transponder bzw. die Spannungsversorgung erfolgt mittels Radiowellen die das Lesegerät aussendet [Fin08]. Die Auswahl der Übertragungsfrequenz ist dabei abhängig von den technischen Anforderungen und den gesetzlichen Regelungen. Mit zunehmender Frequenz erhöht sich auch die Datenübertragungsrate pro Zeiteinheit [Ker07].
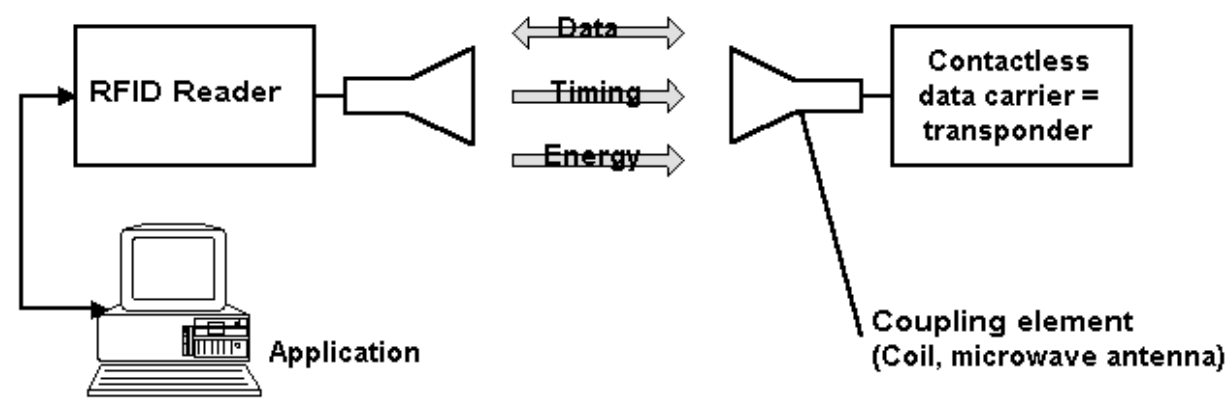

Abbildung 2: RFID Funktionsprinzip [Fin08]

In [KEQ ${ }^{+} 08$ ] und [GG06] werden Anwendungen der Siebdrucktechnologie in der Mess- und Automatisierungstechnik beschrieben. Diese Technologie macht es möglich einen Bruchsensor direkt auf Stahllamellen zu drucken. Der Druck kann sogar in mehreren Schichten übereinander erfolgen. Mittels eines Gummirakels werden über mit Masken versehene Drucksiebe, silberhaltige Metallpasten und 
Dielektrika in einem Multilayeraufbau auf das entsprechende Bauteil gedruckt. Wärmestrahlung oder UV-Licht dienen zum Aushärten der jeweiligen Druckpasten.

\section{RFID-Monitoring-System (RMS)}

Aus bekannten Problemen sowie den Anforderungen der Praxis müssen Monitoring Systeme grundlegende Überwachungsaufgaben wie Messungen und Aufzeichnungen der typischen Versatzarten von Kupplungen erfassen können. Der Abstand des Lesegerätes zum Transponder sollte einen Mindestabstand von $5 \mathrm{~mm}$ nicht unterschreiten. Umgebungstemperaturen von -30 bis $+80{ }^{\circ} \mathrm{C}$ (kurzzeitig bis 120 $\left.{ }^{\circ} \mathrm{C}\right)$ sind im allgemeinen Maschinenbau üblich. Umgebungsmedien können Luft mit geringen Anteilen von mechanischen Abrieb aller Art, Öle und Fette sein.

Stahllamellenkupplungen realisieren eine spielfreie, drehstarre Kraftübertragung auch unter Axial- und Winkelversatz. Kritisch dabei ist der nicht immer zu vermeidende Versatz der beiden zu verbindenden Wellenenden. Das heißt, es kann und darf bei der Neumontage ein definierter Winkelversatz von $0,5^{\circ}$ bzw. 1, $0^{\circ}$ und ein Axialversatz von 0,2 bis $9 \mathrm{~mm}$ je nach Bauart und Größe zulässig sein. Bei diesem Kupplungstyp muss mit Drehzahlen von bis zu 2600U/min in der Standardausführung und maximal bis 6400U/min im ausgewuchteten Zustand gerechnet werden.

Interessant für eine Überwachung sind der Bruch der äußeren Lamelle und die Änderung von Axial- und Winkelversatz bis zu einem vom Hersteller vorgegebenen Grenzwert.

\section{$3.1 \quad$ RFID-Elektronik}

Längst werden RFID Systeme nicht nur zur Erkennung und Erfassung von logistischen Daten sondern auch zur Warenidentifikation und Diebstahlschutz verwendet.

In Anlehnung an die RFID-Technologie und durch die steigenden Anforderungen im Bereich der Energieeffizienz wurden Systeme entwickelt, um ehemals leistungsintensive Sensoren so zu modifizieren, damit diese mit möglichst geringer Energieversorgung dauerhaft, eventuell auch autark, mit dementsprechenden „Energy Harvesting“ zu betreiben. Mit Elementen dieser Technologie sowie aus der Telemetrie stammenden Funkübertragung mit Mikrowellen im Frequenzbereich von 2,4GHz, wurde ein entsprechender Transponder entwickelt. Den Kern der Elektronikentwicklung bilden energieoptimierte Bauteile, die zu einem System vereint und als Einheit immer noch sehr energieeffizient arbeiten (Abbildung 3: Ringförmiges Transponderlayout).

Der Transponder konnte als passive Einheit ausgelegt werden und somit eine völlige Wartungsfreiheit garantieren. Die Energieversorgung erfolgt hierbei durch ein „Remote Coupling" Verfahren [Fin08]. Bei den meisten RFID-Systemen dieser Art, wie auch bei dem entwickelten Sensorsystem, wird eine induktive Kopplung verwendet. Bei dieser Kopplung wird eine Reichweite, bei der die benötigte 
Energiemenge sicher und dauerhaft übertragen werden kann, von einigen mm bzw. $\mathrm{cm}$ angegeben.

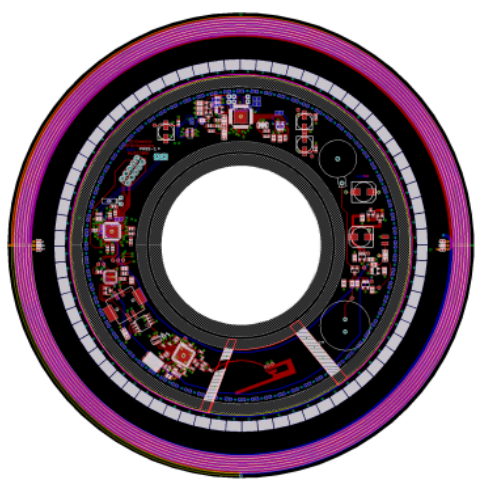

Abbildung 3: Ringförmiges Transponderlayout

Da eine maximale Messrate von $1 \mathrm{kHz}$ und einer Bitzahl von 120 vorgegeben waren, musste die Datenübertragung durch eine kontinuierliche Überwachung mit einer RFFunkübertragung im Mikrowellenbereich erfolgen. Da der ZigBee IEEE 802.15.4 Standard eine optimale Energieeffizienz bei der benötigten Übertragungsrate besitzt ist dieser bevorzugt worden [Kup07]. Der Datentransfer ist als „Point to Point" Strecke aufgebaut. Unnötige Beacons sowie Netzwerkaufbauten wurden mit der Begründung des Energieverbrauchs nicht verwendet.

Da bei höherer Frequenz nicht nur die Datenrate steigt, sondern auch die Störanfälligkeit gegenüber Metallen und Wasser zunimmt, ist das System mit einem softwareseitigen Sicherheitskonzept beaufschlagt worden. Die Übertragung erfolgt Vollduplex d.h. es wird nach jedem Datensatz kontrolliert ob dieser auch empfangen worden ist. Mit dem CRC Verfahren werden zusätzlich Prüfsummen berechnet, um Fehler erkennen zu können [Lan06]. Parallel dazu sind im IEEE 802.15.4 Standard Sicherungssysteme eingebaut, die Übertragungsfehler schon in den unteren Schichten des OSI-Modells erkennen. Sollte ein Fehler auftreten wird der Datensatz erneut gesendet und nach dreimaligem Fehler mit einer Kennung versehen. Während dieser Datenübermittlung werden die anlaufenden Daten in einem FIFO Register gespeichert. Da eine konstante Messrate vorgegeben ist, sind die Daten am Lesegerät dennoch eindeutig einer gewissen Zeit zuzuordnen. Diese Übertragungstechnik benötigt im Gegensatz zum Backscatter oder Lastmodulation enorm viel Energie [Fin08][ Ker07]. Um dieses zu bewältigen wurde ein entsprechend großer Energiespeicher implementiert, der selbst beim Ausfall der Energiezufuhr noch einige Minuten den Datentransfer aufrecht erhalten kann.

\subsection{Sensoren}

Stahllamellenkupplungen werden zahlreichen Belastungsarten währen ihrer Verwendung ausgesetzt. Die Übertragung von Drehmoment als Hauptfunktion und die dabei möglichen Betriebsbedingungen wie Winkelversatz, Radialversatz, Axialversatz, Drehmoment und Stoßbelastungen stellen eine große Herausforderung 
an das Material und die angrenzenden Bauteile dar. Zum Messen des jeweiligen Verformungszustandes wurden Dehnungsmessstreifen belastungsspezifisch auf die Stahllamelle positioniert. Mit Hilfe einer FEM-Simulation konnte das Verhalten des Lamellenpaketes unter extremen Einsatzbedingungen nachgebildet werden. Vor allem durch die Auswertung der Hauptspannungsvektoren bei unterschiedlichen Betriebszuständen konnte eine genaue Position bzw. Ausrichtung und Anzahl der notwendigen Dehnungsmessstreifen theoretisch ermittelt werden. An kombinierten Lastfällen konnte die Art der Verformung und der Spannungsverlauf der Vergleichsspannung nach Mises anhand des Stahllamellenpaketes visualisiert werden (Abbildung 4: FEM-Analyse Stahllamelle).

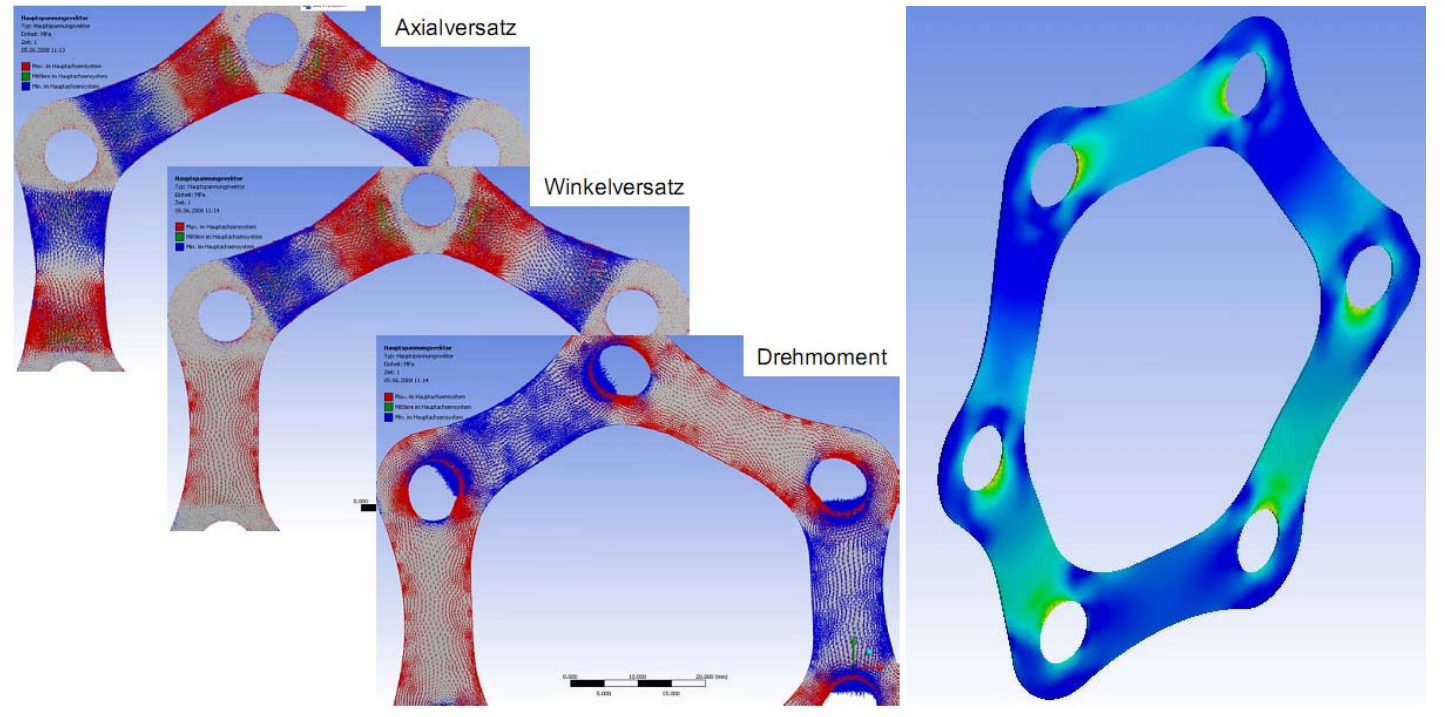

Abbildung 4: FEM-Analyse Stahllamelle

Die FEM-Analyse lieferte auch mögliche Positionen und Gestaltungsmöglichkeiten des Bruchsensors für die Stahllamelle.

Ein mehrschichtiger Siebdruckaufbau direkt auf der Stahllamelle, bestehend aus UV aushärtenden Dielektrikum, Silberleitlack und UV Dielektrikum unter Verwendung von speziell angefertigten Drucksieben und eines Handrakels, konnten erste positive Ergebnisse in dynamischen Dauerversuchen verzeichnet werden (Abbildung 5: 3Schicht-Siebdruck). 


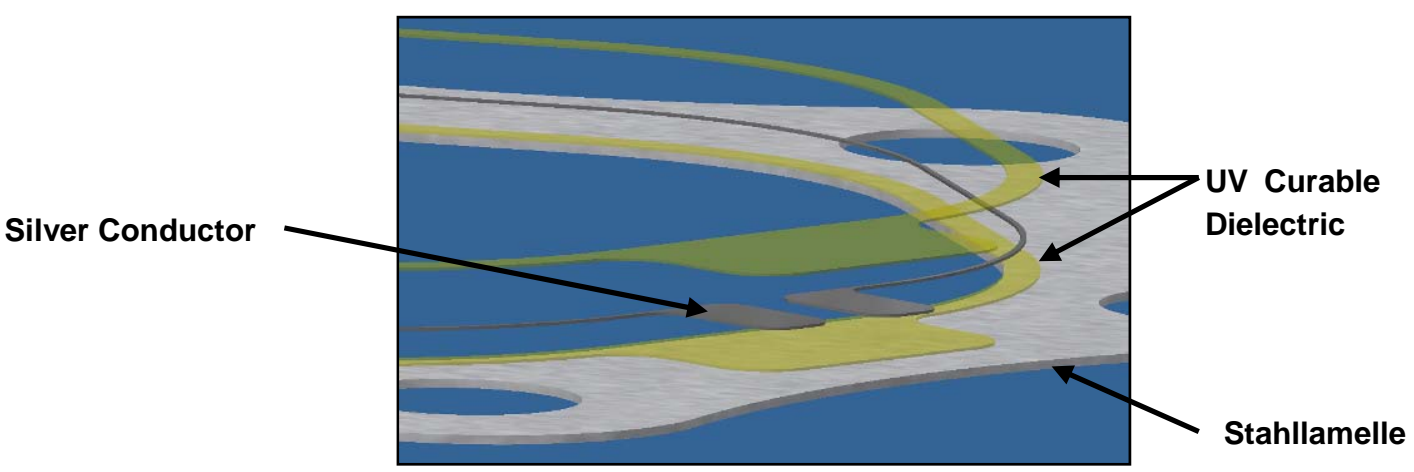

Abbildung 5: 3-Schicht-Siebdruck

Die Position und die Anzahl der notwendigen Dehnungsmessstreifen wurden durch zahlreiche Tests verifiziert. Am finalen Prototypen konnte mit nur einer Messstelle der geforderte Versatz ermittelt werden (Abbildung 6: Sensorlamelle).

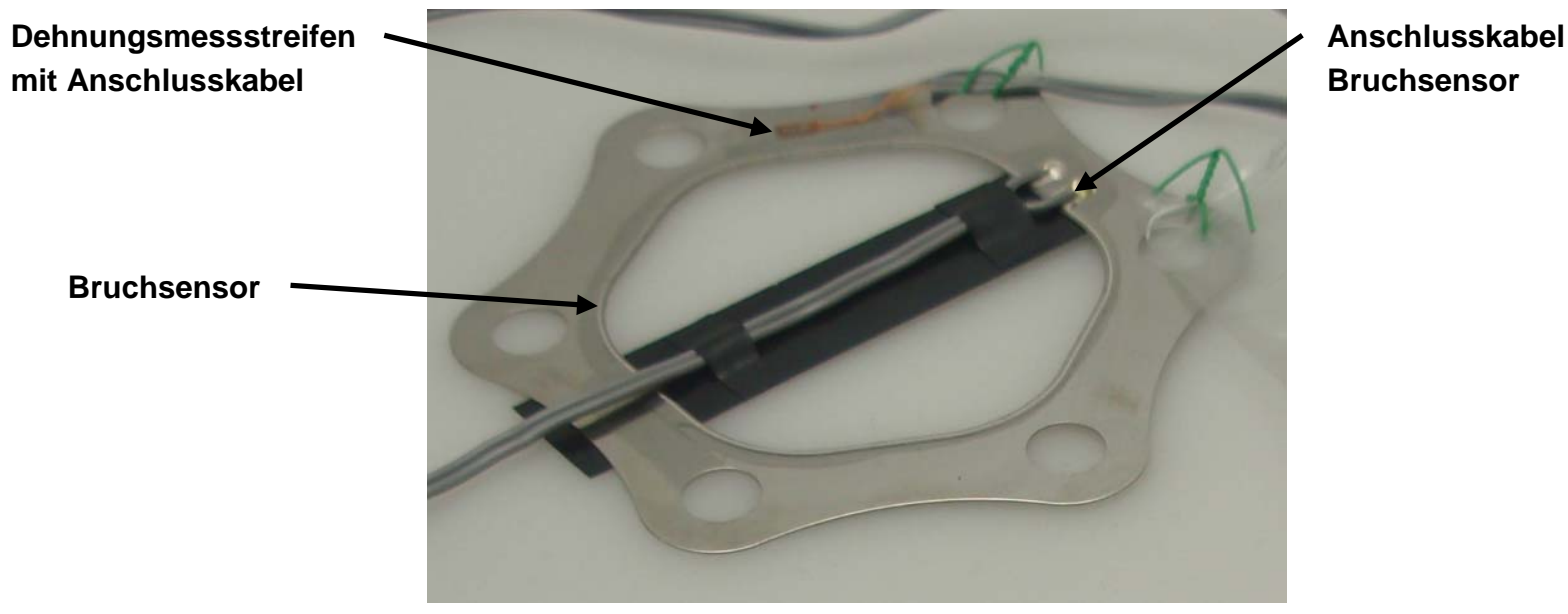

Abbildung 6: Sensorlamelle

\subsection{Prototyp}

Aufbauend auf den Grundlagen entstanden Prototypen des RFIDÜberwachungssystems (Abbildung 7: RFID-Monitoring-System (RMS), Prototyp). Positiv wirkte sich beim Einsatz des Systems die systemeigene Überwachungssoftware aus, welche eine komfortable Bedienung und Auswertung der Messwerte erst ermöglichte. 


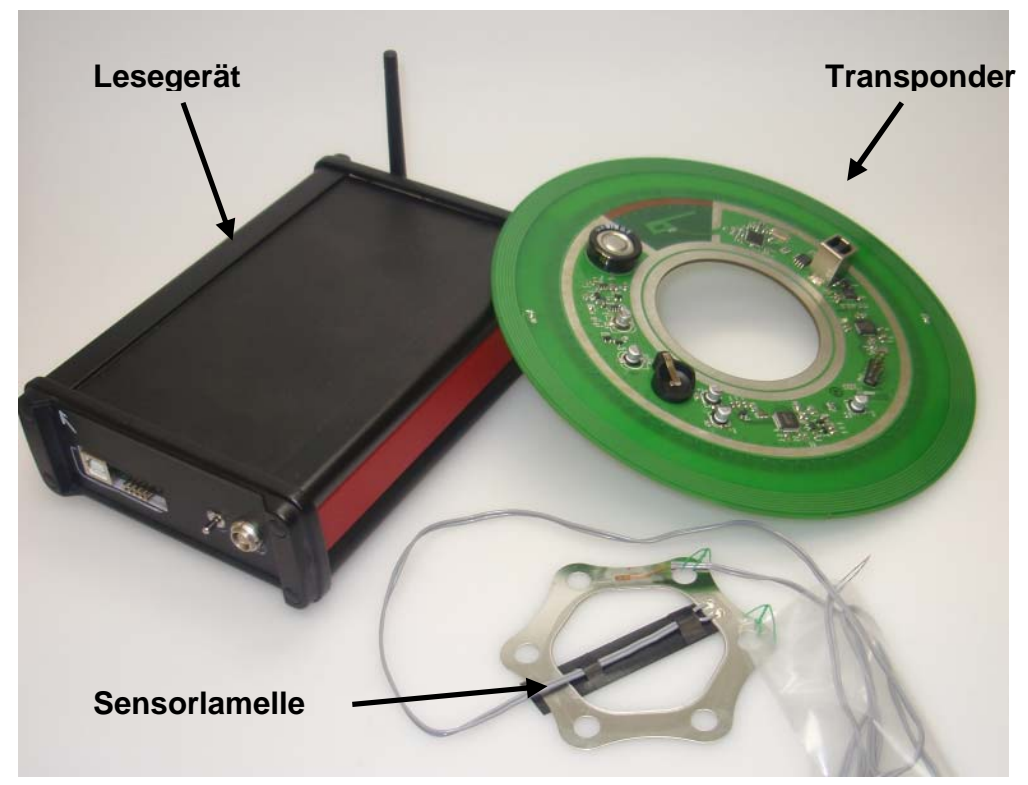

Abbildung 7: RFID-Monitoring-System (RMS), Prototyp

Den prinzipiellen Aufbau und die Funktionsweise des RFID Überwachungssystems wird im folgendem Blockschaubild verdeutlicht (Abbildung 8: Blockschaubild RFIDÜberwachungssystem).

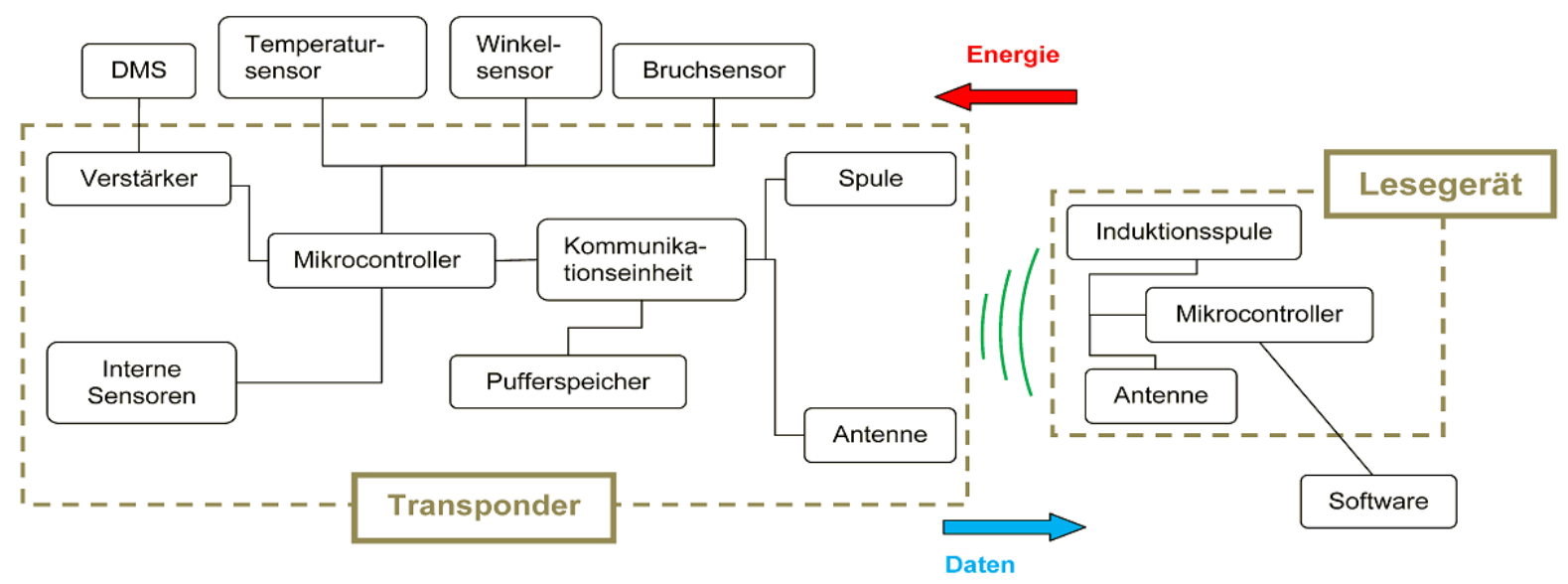

Abbildung 8: Blockschaubild RFID-Überwachungssystem

\section{Experimentelle Untersuchungen}

Ein Praxistest des RFID-Überwachungssystems erfolgte an einer doppelkardanischen Stahllamellenkupplung der Bauform SX 90-6. Beide Lamellenpakete wurden jeweils mit zwei Sensorlamellen und einem Transponder bestückt. Das Auslesen dieser Systeme erfolgte separat über zwei Lesegeräte (Abbildung 9: RMS Prototyp im Testeinsatz). 


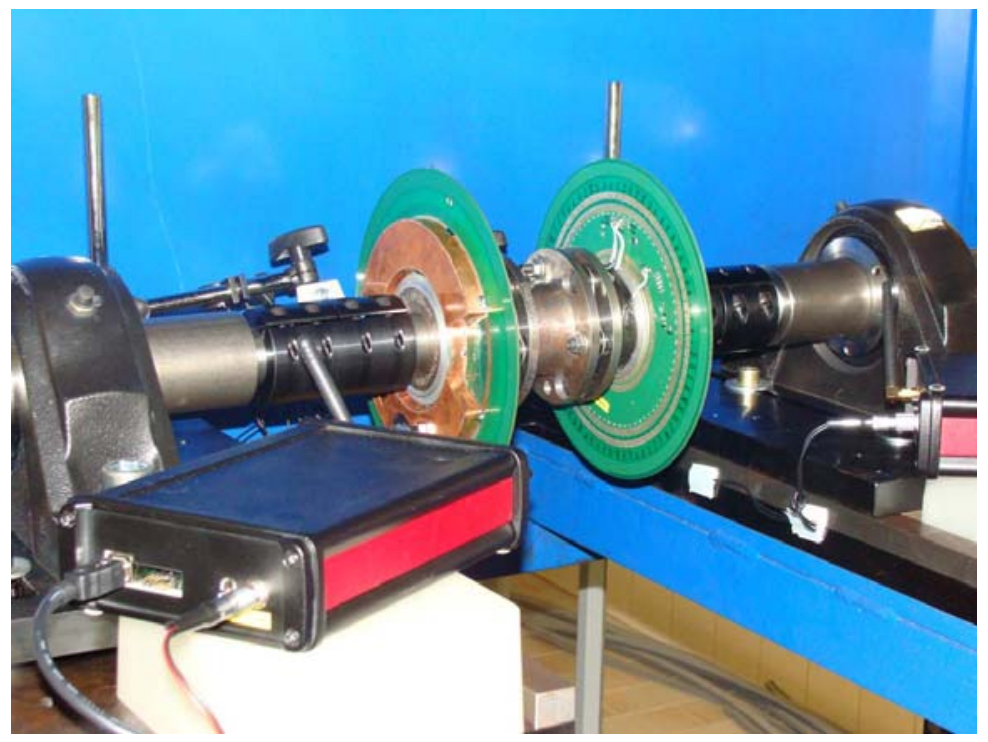

Abbildung 9: RMS Prototyp im Testeinsatz

Ein aus 70 Einzelversuchen bestehende Versuchsprogramm diente zur Ermittlung aller relevanten Kupplungszustände, wie Winkelversatz, Axialversatz und Drehmoment, einzeln und in Kombination untereinander. Für jeden einzelnen Betriebszustand wurden drei Messungen an jedem der zwei Lamellenpakete durchgeführt, wobei die jeweils äußeren Lamellen eines jeden Lamellenpaketes mit identisch angeordneten Dehnungsmessstreifen bestückt waren. Durch eine statistische Auswertung über die Vielzahl der Messungen konnten Messfehler minimiert werden.

Während des Dauerlaufes wurde die Kupplung zyklisch dynamisch und statisch belastet. Im gesamten Testzeitraum war das Überwachungssystem durchgängig in Betrieb und lieferte in Minutenabständen den gemessenen Kupplungszustand. Gegen Ende des Testzeitraumes wurde der Winkelversatz stetig erhöht, um den Bruch aller Lamellen herbeizuführen. Somit konnte überprüft werden, ob das RFID Überwachungssystem auch einen Bruch der Lamellen sicher detektieren kann.

Eine Aufbereitung der gemessenen Rohdaten erfolgte durch die Verwendung eines Hochpassfilters. Die Mittelung aller positiven und negativen Amplituden der gemessenen Schwingungen bildete die Grundlage der Hüllkurvendarstellungen aller relevanten Belastungssituationen. Anhand dieser Hüllkurven konnte der aktuelle Winkelversatz des überwachten Kupplungssystems abgeleitet werden. (Abbildung 10: Winkelversatz gemessen). Information über den Zustand der äußeren Lamellen hinsichtlich eines Bruchs wurden ebenfalls bereitgestellt. 


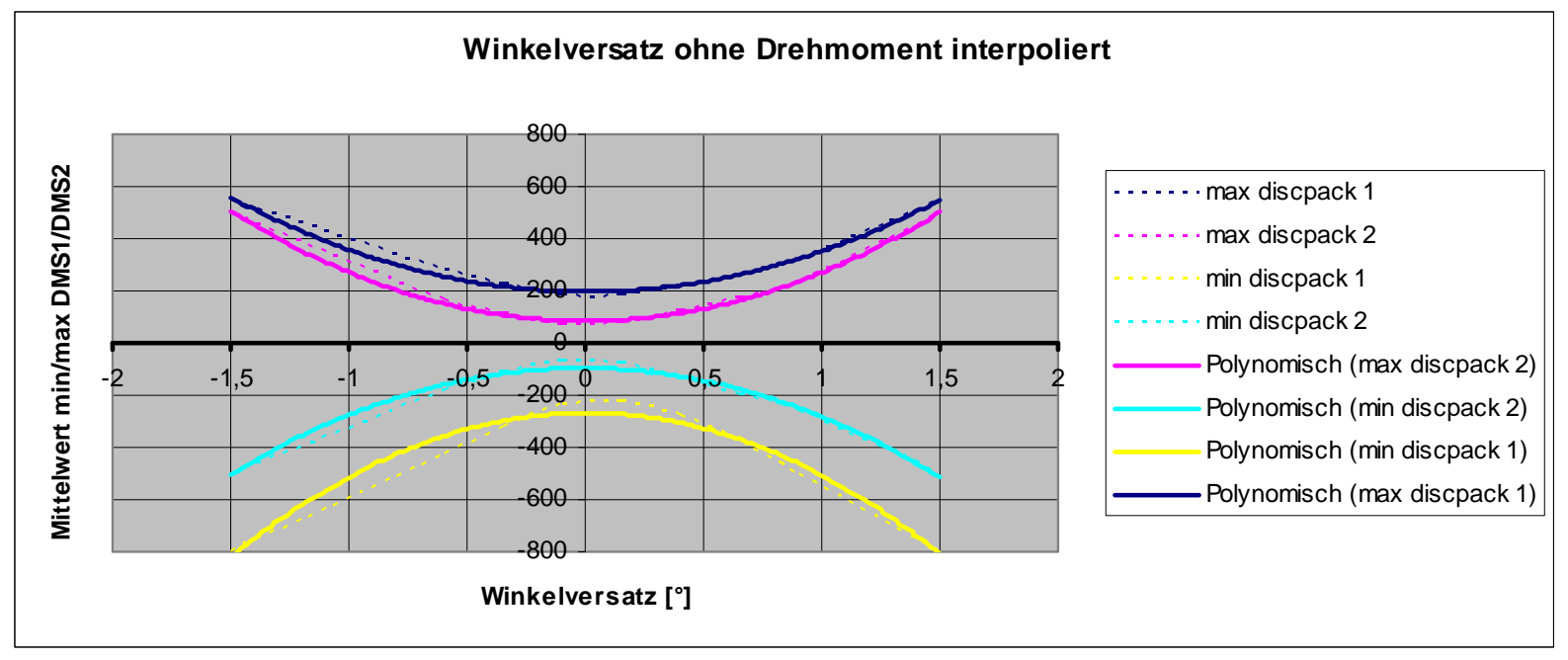

Abbildung 10: Winkelversatz gemessen

Im Ergebnis konnten Erfahrungen zur Systemüberwachung über einen längeren Zeitraum gesammelt und somit die Standfestigkeit nachgewiesen werden. Zudem wurde der Nachweis erbracht, dass das Überwachungssystem nicht nur den kritischen Wellenversatz erkennt, sondern auch einen Lamellenbruch sicher diagnostizieren kann.

Aus der Entwicklung und den Tests konnten folgende technische Parameter für das Überwachungssystem abgeleitet werden:

- Netzspannung: 220V

- Systemspannung: $12 \mathrm{~V}$ DC 0,5A

- Umgebungstemperatur: $-20 \ldots+80^{\circ} \mathrm{C}$

- Abtastfrequenz: $300 \mathrm{~Hz}$

- Abstand Lesegerät / Transponder: bis zu 1m

- Drehwinkelsensor: mechanisch auf Transponder

- Drehwinkelgenauigkeit: $>2^{\circ}$

- Temperaturmessung: von $-20 \ldots+300^{\circ} \mathrm{C}$ im Transponder integriert

- Externe Anschlüsse:

$$
\begin{aligned}
& -4 \text { DMS } \\
& -2 \text { Bruchsensoren }
\end{aligned}
$$

\section{$5 \quad$ Zusammenfassung}

Das entwickelte Überwachungssystem für rotierende Maschinenelemente (RMS), auf Grundlage der RFID Technik, eröffnet für den industriellen Anwender ein enormes wirtschaftliches Potential hinsichtlich Wartung und Instandhaltung von Anlagen. 
Wartungsarbeiten können besser geplant und teure Stillstandzeiten oder sogar Folgeschäden vermieden werden.

Ein Prototypensystem wurde erfolgreich an einer doppelkardanischen Stahllamellenkupplung ausführlich getestet. Zum Testumfang zählten nicht nur Grundlagentests zur Funkübertragung und Sensoreinstellung, sondern auch ausführliche Dauerläufe bis zum Bruch der Lamellen. Aufgezeichnet und ausgewertet wurden dabei die Messwerte mit der eigens dafür entwickelten Überwachungssoftware.

Die Ergebnisse können auch auf Überwachungssysteme für andere Baugrößen oder Maschinenelemente, wie Rutschnaben oder Rutschkupplungen, übertragen werden. Dabei ist es auch möglich, durch die Verwendung anderer Sensoren, weitere Zustandsdaten, wie Temperatur oder Schlupfwinkel, zu überwachen.

\section{Literatur}

[Fin08] Finkenzeller, Klaus: RFID-Handbuch, 4. Auflage. München: Carl Hanser Verlag, 2008.

[GG06] Gevatter, Hans-Jürgen; Grünhaupt, Ulrich: Handbuch der Mess- und Automatisierungstechnik im Automobil, 2. vollst. bearb. Auflage. Berlin Heidelberg: Springer-Verlag, 2006.

[KEQ ${ }^{+}$08] Kallenbach, Eberhard; Eick, Rüdiger; Quent, Peer; Ströhla, Tom; Feindt, Karsten: Elektromagnete, 3. Auflage. Wiesbaden: Vieweg + Teubner, 2008.

[Ker07] Kern, Christian: Anwendung von RFID-Systemen, 2. verb. Auflage. Berlin: Springer Verlag, 2007.

[Kup07] Kupris, Gerald: Zigbee Datenfunk mit IEEE 802.15.4, 1. Auflage. Poing: Franzis Verlag, 2007.

[Lan06] Lang, Hans Werner: Algorithmen in Java, 2. Auflage. München: Oldenbourg Wissenschaftsverlag, 2006.

[Lov10] Lovejoy Inc.: Disc Catalog, 2010. 